\title{
Bifurcation analysis of a first time-delay chaotic system
}

\author{
Tianzeng $\mathrm{Li}^{1,2^{*}}$, Yu Wang ${ }^{1,3}$ and Xiaofeng Zhou ${ }^{4}$
}

\section{"Correspondence:}

litianzeng27@163.com

'School of Mathematics and

Statistics, Sichuan University of

Science and Engineering, Zigong,

China

${ }^{2}$ Artificial Intelligence Key

Laboratory of Sichuan Province,

Zigong, China

Full list of author information is

available at the end of the article

\begin{abstract}
This paper deals with the dynamic behavior of the chaotic nonlinear time delay systems of general form $\dot{x}(t)=g(x(t), x(t-\tau))$. We carry out stability analysis to identify the parameter zone for which the system shows a stable equilibrium response. Through the bifurcation analysis, we establish that the system shows a stable limit cycle through supercritical Hopf bifurcation beyond certain values of delay and parameters. Next, a numerical simulation of the prototype system is used to show that the system has different behaviors: stability, periodicity and chaos with the variation of delay and other parameters, which demonstrates the validity of our method. We give the single- and two-parameter bifurcation diagrams which are employed to explore the dynamics of the system over the whole parameter space.
\end{abstract}

Keywords: Bifurcation analysis; Periodicity; Chaos; Hopf bifurcation

\section{Introduction}

For the last decades, time-delayed dynamical systems have been attracting the attention of researchers of various fields, including mathematics, biology, economics, physics, engineering, etc. [1-4]. Many natural systems are mathematically modeled by nonlinear delay differential equations which contain one or more time delays. Successful examples include blood production in patients with leukemia [5], dynamics of optical systems [6, 7], population dynamics [8], physiological model [9], El Niño/southern oscillation [10], the Lorentz force with Liénard-Weichert potentials [11], neural network with three neurons [12], delay feedback control and synchronization $[13,14]$, etc. The presence of a delay in a system makes the system infinite-dimensional, and may lead to an unstable and oscillatory response. In particular, the time delay of a nonlinear system may give rise to various complex phenomena such as bifurcation, chaos, hyperchaos, multistability, etc.

There are many reasons why the nonlinear delay dynamical systems have been studied in the mathematical modeling of the naturally occurring phenomena $[15,16]$. Firstly, the delay differential equations show higher-dimensional chaotic behavior which cannot be anticipated by a low-dimensional system [17]. It is important to understand and explore the behavior of these systems both from the academic and engineering perspective. Secondly, infinite dimensionality of delayed systems offers a great opportunity to the researchers to harness the richness of hyperchaos, having multiple positive Lyapunov exponents. It has been proved that communication with a low-dimensional chaos is not fully secure because an eavesdropper can reconstruct chaotic attractors and retrieve a hidden

(c) The Author(s) 2019. This article is distributed under the terms of the Creative Commons Attribution 4.0 International License (http://creativecommons.org/licenses/by/4.0/), which permits unrestricted use, distribution, and reproduction in any medium, provided you give appropriate credit to the original author(s) and the source, provide a link to the Creative Commons license, and indicate if changes were made. 
message $[18,19]$. Therefore, the synchronization of hyperchaotic systems has been proposed as an alternative method for improving the security in the communication schemes [20-22]. It has been proved that a simple time delay system with suitable nonlinearity can produce a hyperchaotic signal with multiple positive Lyapunov exponents, thus making it a good candidate for a secure communication system [23, 24]. Beside a secure communication system, chaotic and hyperchaotic systems have important applications in chaos-based noise generators [25], improvement of motion capabilities and of sensors in robotics [26, 27], etc.

Hence, the bifurcation analysis of time-delay chaotic systems has been the subject of worldwide attention in the last decades [28]. In [29], Campbella and Ncubeb gave an explicit description of the region of stability for a linear scalar delay differential equation consisting of two arbitrarily distributed time delays. El-Dessoky et al. investigated the local Hopf bifurcation in Shimizu-Morioka chaotic system with delayed feedback control [30]. Song et al. studied the stability and Hopf bifurcation in a model of gene expression with distributed time delays [31]. In [32], Feng and Wei investigated the effect of delayed feedbacks on the generalized Sprott B system with hidden attractors and its local Hopf bifurcation. Atay and Ruan studied systems of coupled units in a general network configuration with a coupling delay [33]. Yeniceri and Yalcin introduced the first generalization for time-delay sampled-data chaotic system in order to generate multiscroll attractors [34]. Wei et al. made a lot of contributions to the Hopf bifurcation analysis of many equations, such as Mackey-Glass system [35], delayed Nicholson blowflies equation [36], and a neural network model with delay [37]. We would like to mention that there are several papers on the bifurcation formed by a branch of periodic orbits of a family functional differential equations, see Dormaer [38, 39], and the global existence of periodic solutions in delayed differential equations based on the global Hopf bifurcation theory given by Wu [40]; for example, see the works given by Ruan and Wei [41], Song and Wei [42], Wen and Wang [43]. Tang et al. casted light on network synchronous state stability via studying the bifurcation (or transition) problem of network synchronized regions with varying nodal dynamics [44]. Xiao et al. proposed a delayed fractional-order congestion control model which is more accurate than the original integer-order model when depicting the dual congestion control algorithms [45].

In this paper, we first study a general chaotic nonlinear time delay system with a constant time delay. We carry out stability analysis to identify the parameter zone for which the system shows a stable equilibrium response. Through the bifurcation analysis, the system shows a stable limit cycle through supercritical Hopf bifurcation beyond certain values of delay and parameters of the system. Next, a numerical simulation of the prototype system shows that the system shows stability, periodicity and chaos with the variation of delay and other system parameters. We give the single- and two-parameter bifurcation diagrams which are employed to explore the dynamics of the system over the whole parameter space. The numerical results demonstrate the validity of our method.

This paper is organized in the following manner: In Sect. 2, the main results about the stability and bifurcation analysis are discussed. In Sect. 3, the stability analysis of the prototype model is presented. In Sect. 4, a numerical simulation of the system shows that with the variation of delay and other system parameters, the system shows stability, periodicity and chaos. Finally, conclusions are given in Sect. 5. 


\section{Stability and bifurcation analysis}

\subsection{The system description}

For a discussion of the stability analysis, we consider the following delay differential equation:

$$
\dot{x}(t)=g(x(t), x(t-\tau)) \quad\left(t \geq t_{0}\right)
$$

where $\tau \geq 0$ is the time delay, and $g$ is a function of $x(t)$ and $x(t-\tau)$. Equation (1) is of very general nature because of the function $g$, which can be linear, piecewise linear, or nonlinear. A few particular cases of this system are Mackey-Glass equation $\dot{x}(t)=-b x+$ $a x_{\tau} /\left(1+x_{\tau}^{c}\right)$ arising in the model of leukemia [46], Ikeda equation $\dot{x}(t)=b x(t)+a \sin \left(c x_{\tau}\right)$ in optics [47], and the piecewise linear Lu-He model $\dot{x}(t)=-a x(t)+b f\left(x_{\tau}\right)$ [48], where $f(x)$ is a piecewise linear function. Here $t_{0}$ is the initial interval and $\tau>0$ corresponds to the delay time, which represents the time interval between the start of an event at one point and its resulting action at another point in the system. The solution of the system equation defined in (1) is determined uniquely when an initial function $\phi(t)$ defined on an initial interval is prescribed as

$$
x(t)=\phi(t), \quad \text { for } t \in\left[t_{0}-\tau, t_{0}\right] .
$$

The time delay is approximated or its effects are generally ignored by modelers. Several researches have commented on the dangers modelers risk if they ignore delays which they think are small [49]. In the case of approximation used for the delay elements, their effects on the system behavior can only be partly observed. The delay elements arise naturally in various diverse fields: chemical kinetics, infectious diseases, and the navigational control of ships and aircraft [49]. Therefore, the subject of differential equations with time delay is now a rapidly growing field, and numerical solution of systems modeled by delay differential equations is both of theoretical and practical interest. The bibliography prepared by Baker et al. shows recent developments and interests in this field [50].

In this paper, we first discuss the stability and bifurcation analysis of the above nonlinear continuous delay system (1). Through the bifurcation analysis, we establish that the system shows a stable limit cycle through supercritical Hopf bifurcation beyond certain values of delay and system parameters. Next, a numerical simulation of the considered nonlinear system $[51,52]$ is used to show that the system exhibits stability, periodicity and chaos with the variation of delay and other system parameters, which demonstrates the validity of the proposed theory. The nonlinear continuous system in dimensionless form with one-state variable is as follows:

$$
\dot{x}(t)=\gamma x(t-\tau)-\beta f(x(t-\tau)), \quad\left(t \geq t_{0}\right),
$$

where $\gamma$ and $\tau$ are positive parameters of the system. In [51], the author uses numerical simulation to study the dynamical behavior of the system with the variation of the system parameters. In this paper, we will carry out bifurcation analysis to study the rich dynamical behavior of the system in theory, and we will use a numerical simulation of the system to show that with the variation of delay and other system parameters, the system shows stability, periodicity and chaos. 


\subsection{Stability analysis of delay differential equation}

Consider the delay differential equation (1), namely $\dot{x}(t)=g(x(t), x(t-\tau))$. The equilibrium points $x^{*}$ are obtained by solving

$$
g\left(x^{*}, x^{*}\right)=0 \text {. }
$$

Then, we consider the linearization of the system near the equilibrium $x^{*}$. Defining a small perturbation $\xi=x-x^{*}$ to equilibrium solution and using Taylor's approximation, we get a linearized equation of (1) as

$$
\dot{\xi}=a \xi+b \xi_{\tau},
$$

where $a=\left.J_{0}\right|_{x^{*}}=\left.\frac{\partial g\left(x(t), x_{\tau}\right)}{\partial x}\right|_{x^{*}}, b=\left.J_{\tau}\right|_{x^{*}}=\left.\frac{\partial g\left(x(t), x_{\tau}\right)}{\partial x_{\tau}}\right|_{x^{*}}$. Using the Laplace transform, the characteristic equation can be obtained as

$$
\lambda=a+b e^{-\lambda \tau} .
$$

As we known, an equilibrium point $x^{*}$ is asymptotically stable if all roots $\lambda_{i}$ of the characteristic equation (6) satisfy

$$
\operatorname{Re}\left(\lambda_{i}\right)<0, \quad \forall i
$$

If the delay time $\tau=0$, the eigenvalue is $\lambda=a+b$. Condition (7) then takes the form $a+b<$ 0 , which implies that the equilibrium point $x^{*}$ is stable. In the following we consider the delay time $\tau \neq 0$. Hopf bifurcation will appear if at least one of the eigenvalues crosses the imaginary axis from the left and enters the right half-plane. Putting $\lambda=\mu+i \omega$, if $\mu$ varies from the left to right, we can say that $\mu<0$ is a stable state, $\mu>0$ is a bifurcated state, and $\mu=0$ is the limiting case. At the emergence of Hopf bifurcation, we put $\mu=0$. Thus using $\lambda=i \omega$, we have

$$
i \omega=a+b e^{-i \omega \tau}
$$

Now equating the real and imaginary parts on both sides of the above equation, we obtain

$$
\left\{\begin{array}{l}
a=-b \cos (\omega \tau) \\
\omega=-b \sin (\omega \tau) .
\end{array}\right.
$$

This implies $\omega= \pm \sqrt{b^{2}-a^{2}}$, and the sets of solutions of $\tau$ are as follows:

$$
\tau_{k}= \begin{cases}\arccos \left(-\frac{a}{b}\right)+2 k \pi, & \text { for } \omega>0 \\ 2 \pi-\arccos \left(-\frac{a}{b}\right)+2 k \pi, & \text { for } \omega<0\end{cases}
$$

where $k=0,1,2, \ldots$. And if we set

$$
\omega_{0}=\sqrt{b^{2}-a^{2}}
$$


then $\lambda_{k}(\tau)=\mu_{k}(\tau)+i \omega_{k}(\tau)$ is a root of (6) satisfying $\mu_{\tau_{k}}=0$ and $\omega_{k}\left(\tau_{k}\right)=\omega_{0}$. We have the following results.

Lemma $1 \mu_{k}^{\prime}\left(\tau_{k}\right)>0$.

Proof Differentiating both sides of (6) with respect to $\tau$, it follows that

$$
\frac{d \lambda}{d \tau}=b e^{-\lambda \tau}\left(-\frac{d \lambda}{d \tau}-\lambda\right)
$$

Therefore, noting that $\lambda=a+b e^{-\lambda \tau}$, we obtain

$$
\frac{d \lambda}{d \tau}=-\frac{\lambda(\lambda-a)}{1+\tau(\lambda-a)}
$$

and

$$
\left.\frac{d \lambda}{d \tau}\right|_{\tau=\tau_{k}}=-\frac{i \omega_{0}(i \omega-a)}{1+\tau(i \omega-a)}=\frac{\omega_{0}^{2}-i\left(\omega_{0} a-\omega_{0} \tau a^{2}-\omega^{3} \tau\right)}{(1-\tau a)^{2}+\omega_{0}^{2} \tau^{2}} .
$$

This implies that

$$
\mu_{k}^{\prime}\left(\tau_{k}\right)=\frac{\omega_{0}^{2}}{(1-\tau a)^{2}+\omega_{0}^{2} \tau^{2}}>0
$$

Hence, $\frac{d \mu}{d \tau}>0$ on each of the critical surfaces $\tau_{k}$, which implies that there does not exist any eigenvalue with a negative real part across the critical surface. Thus, there is only one possible stability region (under the condition $a+b<0$ ) enclosed by $\tau=0$ and the critical surface $\tau_{0}$.

Then, we have the following main conclusion:

Case I. If $a+b<0$ then $\tau=0$ gives stable solutions. Further, the stability surfaces exist if

$$
b^{2}>a^{2} \text {. }
$$

The stability region is bounded by the plane $\tau=0$ and the closest critical surface $\tau_{0}$.

If condition (16) is not satisfied, then the stability properties will not change in this region $a+b<0$, i.e., the solutions are stable for any $\tau \geq 0$.

Case II. If $a+b>0$ then the system will be unstable for any $\tau \geq 0$ and for any parameter values.

We summarize these conclusions in the following theorem.

Theorem 1 Suppose $x^{*}$ is an equilibrium solution of the delay differential equation (1), namely $\dot{x}(t)=g(x(t), x(t-\tau))$, and $a=\left.J_{0}\right|_{x^{*}}, b=\left.J_{1}\right|_{x^{*}}$. Then we have:

1. If $b \in(-\infty,-|a|)$ then the stability region of $x^{*}$ in $(\tau, a, b)$ parameter space is $\left[0, \tau_{0}\right)$. And if $\tau \in\left(\tau_{k}, \tau_{k+1}\right)$, equation has $2(k+1)$ roots with positive real parts. The equation undergoes Hopf bifurcation at $\tau_{k}, k=0,1,2, \ldots$.

2. If $b \in(-a,+\infty)$ then $x^{*}$ is unstable for any $\tau \geq 0$.

3. If $b \in(a,-a)$ and $a<0$ then $x^{*}$ is stable for any $\tau \geq 0$. 


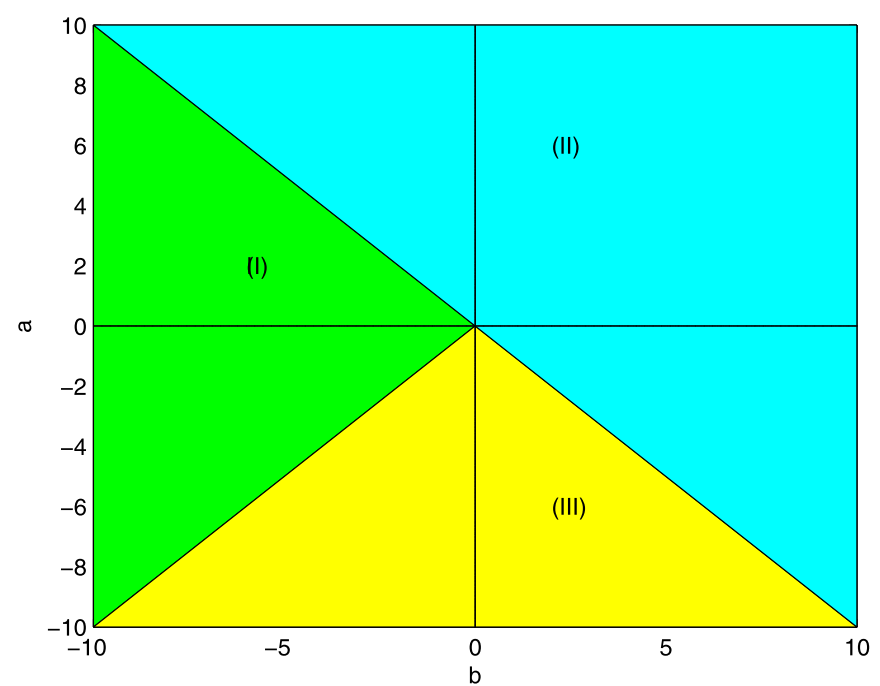

Figure 1 Stability regions of Eq. (1): (I) stable region for the delay in the interval $\left[0, \tau_{1}(0)\right)$, (II) unstable region, (III) stable region

Proof Claim 1. It is followed from $b \in(-\infty,-|a|)$ that $a+b<0$ and $b^{2}>a^{2}$. The equation has a pair imaginary roots $\pm i \omega_{0}$ when $\tau=\tau_{k}(k=0,1,2, \ldots)$. If $\tau \in\left[0, \tau_{0}\right)$, all the roots of equation (1) have negative real parts, i.e., the equilibrium $x^{*}$ is stable. The statement on the number of eigenvalues with positive real parts follows from Lemma 1 and Rouchés theorem [53].

Claim 2. It follows from $b \in(-a,+\infty)$ that $a+b>0$, and then the equation has at least one root with a positive real part for all $\tau \geq 0$, i.e., for equation (1), the equilibrium point $x^{*}$ is unstable for all $\tau \geq 0$.

Claim 3. It follows from $b \in(a,-a)$ that $a+b<0$ and $b^{2}<a^{2}$. For all $\tau \geq 0$, all the roots of the equation have negative real parts, i.e., the equilibrium $x^{*}$ is stable.

These results are also summarized in Fig. 1.

\section{Stability analysis of the prototype model}

The system given in (3) has been used as a prototype model to observe self-oscillations in the shipbuilding industry [54]. In [51], the author studies the basic chaotic behavior by numerical simulation. For theoretically analyzing the complexity of the system given by (1), we rewrite (3) in the following form [52]:

$$
\dot{x}(t)=g\left(x(t), x_{\tau}\right)=\gamma x(t-\tau)-\beta x^{3}(t-\tau),
$$

where $\gamma$ and $\beta$ are positive parameters of the system and $\tau$ is the time delay. The equilibrium condition implies $\dot{x}=0$ and $x(t)=x(t-\tau)=x^{*}$, i.e., $g\left(x^{*}, x^{*}\right)=\gamma x^{*}-\beta x^{* 3}=0$. This implies that the system has three equilibrium points, namely the origin $E_{1}=0$ and $E_{2,3}= \pm \sqrt{\gamma / \beta}$. Based on the analysis of $[40,55,56]$, we will find the value of $\tau$ where the fixed point losses its stability through Hopf bifurcation. From (17), one has

$$
J_{0}=\frac{\partial g\left(x(t), x_{\tau}\right)}{\partial x}=0
$$


and

$$
J_{\tau}=\frac{\partial g\left(x(t), x_{\tau}\right)}{\partial x_{\tau}}=\gamma-3 \beta x^{2}(t-\tau) \text {. }
$$

In the following, our aim is to examine the stability of every equilibrium point.

\subsection{Stability of the equilibrium point $E_{1}$}

In this section, we consider the stability of the equilibrium point $E_{1}=0$. Since we have $\left.J_{0}\right|_{E_{1}}=0$ and $\left.J_{\tau}\right|_{E_{1}}=\gamma$, the characteristic equation is given by

$$
J_{0}+J_{\tau} e^{-\lambda \tau}-\lambda=\gamma e^{-\lambda \tau}-\lambda=0
$$

which implies

$$
\lambda=\gamma e^{-\lambda \tau}
$$

If the delay time $\tau=0$, the eigenvalue $\lambda=\gamma>0$, which implies that the equilibrium point $E_{1}=0$ is unstable. If $\tau \neq 0$, the conclusion can be obtained by Theorem 1 as follows:

Theorem 2 Equation (21) has at least one root with a positive real part for all $\tau \geq 0$, i.e., for equation (17), the equilibrium point $E_{1}=0$ is unstable for all $\tau \geq 0$.

Proof Since $a=\left.J_{0}\right|_{E_{1}}=0$ and $b=\left.J_{1}\right|_{E_{1}}=\gamma>0$, we have $a+b=\gamma>0$, which implies that equation (21) has at least one root with a positive real part for all $\tau>0$. Hence, the equilibrium point $E_{1}=0$ is unstable for $\tau \geq 0$.

\subsection{Stability of the equilibrium points $E_{2,3}$}

In this section, we study the stability of the equilibrium points $E_{2,3}= \pm \sqrt{\gamma / \beta}$ by the same method. Since we have $\left.J_{0}\right|_{E_{2,3}}=0$ and $\left.J_{\tau}\right|_{E_{2,3}}=\gamma-3 \beta \frac{\gamma}{\beta}=-2 \gamma$, the characteristic equation is given by

$$
J_{0}+J_{\tau} e^{-\lambda \tau}-\lambda=-2 \gamma e^{-\lambda \tau}-\lambda=0,
$$

which implies

$$
\lambda=-2 \gamma e^{-\lambda \tau}
$$

If the delay time $\tau=0$, the eigenvalue $\lambda=-2 \gamma<0$, which implies that the equilibrium points $E_{2,3}= \pm \sqrt{\gamma / \beta}$ are stable. In the following, we consider the delay time $\tau \neq 0$. Hopf bifurcation will appear if at least one of the eigenvalues crosses the imaginary axis from the left and enters the right half-plane. Putting $\lambda=\mu+i \omega$, if $\mu$ varies from the left to right, we can say that $\mu<0$ is a stable state, $\mu>0$ is a bifurcated state, and $\mu=0$ is the limiting case. At the emergence of Hopf bifurcation, we put $\mu=0$, thus using $\lambda=i \omega$, we have

$$
i \omega=-2 \gamma e^{-i \omega \tau}=-2 \gamma(\cos (\omega \tau)-i \sin (\omega \tau)) .
$$


Now equating the real and imaginary parts on both sides of above equation, we obtain

$$
\left\{\begin{array}{l}
2 \gamma \cos (\omega \tau)=0, \\
2 \gamma \sin (\omega \tau)=\omega .
\end{array}\right.
$$

This implies $\omega= \pm 2 \gamma$, and the sets of solutions of $\tau$ are as follows:

$$
\tau_{k 1}= \begin{cases}\frac{2 k \pi+\pi / 2}{\omega}, & \text { for } \omega>0, \\ \frac{2 k \pi+3 \pi / 2}{\omega}, & \text { for } \omega<0,\end{cases}
$$

where $k=0,1,2, \ldots$. If we set

$$
\omega_{0}=2 \gamma,
$$

then $\lambda_{k}(\tau)=\mu_{k}(\tau)+i \omega_{k}(\tau)$ is a root of (23) satisfying $\mu_{\tau_{k}}=0$ and $\omega_{k}\left(\tau_{k}\right)=\omega_{0}$. We have the following results.

Lemma $2 \mu_{k}^{\prime}\left(\tau_{k}\right)>0$.

Proof Differentiating both sides of (23) with respect to $\tau$, it follows that

$$
\frac{d \lambda}{d \tau}=-2 \gamma e^{-\lambda \tau}\left(-\frac{d \lambda}{d \tau}-\lambda\right)
$$

Therefore, noting that $\lambda=-2 \gamma e^{-\lambda \tau}$, we obtain

$$
\frac{d \lambda}{d \tau}=-\frac{\lambda^{2}}{1+\lambda \tau}
$$

and

$$
\left.\frac{d \lambda}{d \tau}\right|_{\tau=\tau_{k}}=\frac{\omega_{0}^{2}}{1+i \omega_{0} \tau}=\frac{\omega_{0}^{2}-i \omega_{0}^{3} \tau}{1+\omega_{0}^{2} \tau^{2}}
$$

This implies that

$$
\mu_{k}^{\prime}\left(\tau_{k}\right)=\frac{\omega_{0}^{2}}{1+\omega_{0}^{2} \tau^{2}}>0
$$

Lemma 3 For the equilibrium points $E_{2,3}$, there exists a sequence of values of $\tau$

$$
0<\tau_{0}<\tau_{1}<\cdots<\tau_{k}<\cdots,
$$

such that (i) equation (23) has a pair imaginary roots $\pm i \omega_{0}$ when $\tau=\tau_{k}(k=0,1,2, \ldots)$, (ii) if $\tau \in\left[0, \tau_{0}\right)$, all the roots of equation (23) have negative real parts, if $\tau=\tau_{0}$, all roots of equation (23) except $\pm i \omega_{0}$ have negative real parts, and if $\tau \in\left(\tau_{k}, \tau_{k+1}\right)$ for $k=0,1,2, \ldots$, equation $(23)$ has $2(k+1)$ roots with positive real parts. 
Proof From (25) and (26), we obtain that equation (23) has purely imaginary roots $\pm i \omega_{0}$ if and only if $\tau=\tau_{k}$. The statement about the number of eigenvalues with positive real parts in (ii) follows from Lemma 2 and Rouchés theorem [53].

Theorem 3 The equilibrium points $E_{2,3}=0$ are asymptotically stable for $\tau \in\left[0, \tau_{0}\right)$ and unstable for $\tau>\tau_{0}$, and equation (17) undergoes a Hopf bifurcation at $E_{2,3}$ when $\tau=\tau_{k}$ for $=0,1,2, \ldots$.

Proof This theorem can be proof by Lemma 3. It also can be obtained by Theorem 1 with $b \in(-\infty,-|a|)$, where $a=0$ and $b=-2 \gamma<0$.

In this section, we have the conclusion: (i) equation (17) is unstable for $\tau \geq 0$ at the equilibrium point $E_{1}$; (ii) equation (17) is asymptotically stable for $\tau \in\left[0, \tau_{0}\right)$ and unstable for $\tau>\tau_{0}$ at the equilibrium points $E_{2,3}$, and equation (17) undergoes a Hopf bifurcation at the equilibrium points $E_{2,3}$ when $\tau=\tau_{k}$ for $=0,1,2, \ldots$

\section{Numerical simulation}

In the previous section, we obtained that the considered equation at $E_{2,3}$ is asymptotically stable for $\tau \in\left[0, \tau_{0}\right)$ and unstable for $\tau>\tau_{0}$, and equation (17) undergoes a Hopf bifurcation at $E_{2,3}$ when $\tau=\tau_{k}$ for $k=0,1,2, \ldots$. In the following, we will give the dynamic behaviors of the system with different parameter $\gamma$ and different time delay $\tau$. And the bifurcation diagrams will be obtained by plotting the local maxima of $x$, excluding a large number of transients. System (17) is solved numerically using the fifth-order Runge-Kutta algorithm with integration step size $h=0.005$ and the constant initial function $\phi(t)=0.1$ for $t \in[-\tau, 0]$.

\subsection{The dynamic behavior with $\boldsymbol{\gamma}=\mathbf{0 . 5}$}

First, we vary the time delay $\tau$, taking $\gamma=0.5$ and $\beta=1$. When $\tau \geq 1.57$, the fixed point loses its stability through Hopf bifurcation, which is in accordance with the analysis of the previous section. The bifurcation diagram is shown in Fig. 2. According to the numerical simulations and Fig. 2, we find that the system is asymptotically stable for $\tau \in[0,1.57)$. The limit cycle of period-1 becomes unstable and a period-2 cycle appears at $\tau=2.54$. Further period doubling occurs at $\tau=2.65$ (period-2 to period-4). Through a period doubling sequence, the system enters into the chaotic region at $\tau=3.1$. At last, the system becomes divergent at $\tau=3.45$. A phase plane representation in the representative $x-d x / d t$ plane for different $\tau$ is shown in Fig. 3, which shows the following characteristics: stability $(\tau=1)$, period-1 $(\tau=1.8)$, period-2 $(\tau=2.6)$, period-4 $(\tau=2.7)$, and chaos $(\tau=3.1$ and $\tau=3.3)$.

\subsection{The dynamic behavior with $\gamma=1$}

Now we study the dynamic behavior of system (2) by varying the time delay $\tau$ and taking $\gamma=1$ and $\beta=1$. When $\tau \geq 0.79$, the fixed point loses its stability through Hopf bifurcation, and the bifurcation diagram is shown in Fig. 4. According to the numerical simulations and Fig. 4, the following conclusions are found. The system is asymptotically stable for $\tau \in[0,0.79)$. The limit cycle of period- 1 becomes unstable and a period- 2 cycle appears at $\tau=1.27$. Further period doubling occurs at $\tau=1.32$ (period- 2 to period-4). Through a period doubling sequence, the system enters into the chaotic region at $\tau=1.52$. At last, the 


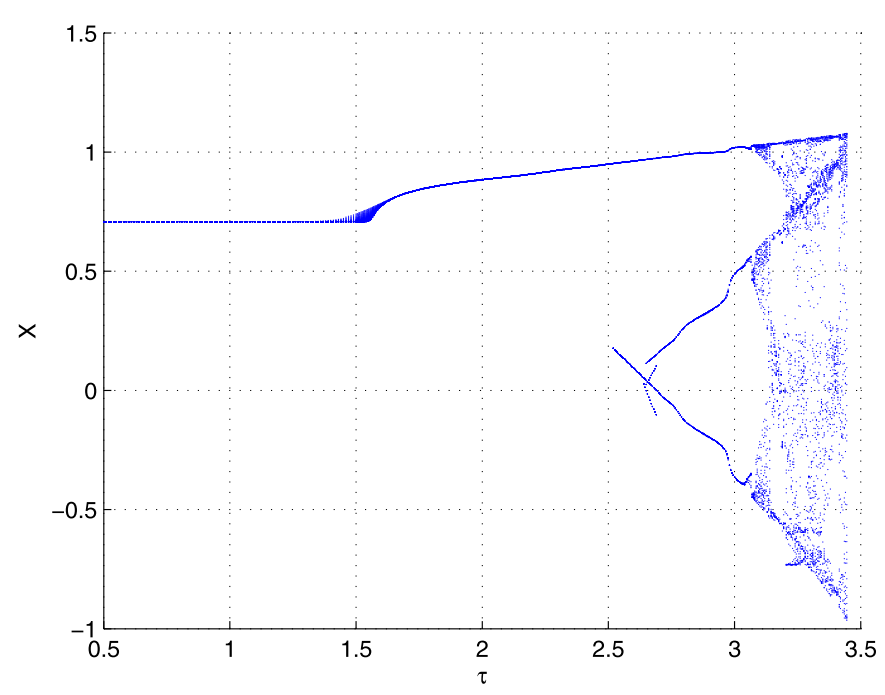

Figure 2 The bifurcation diagram of system (17) with the parameter $\gamma=0.5$
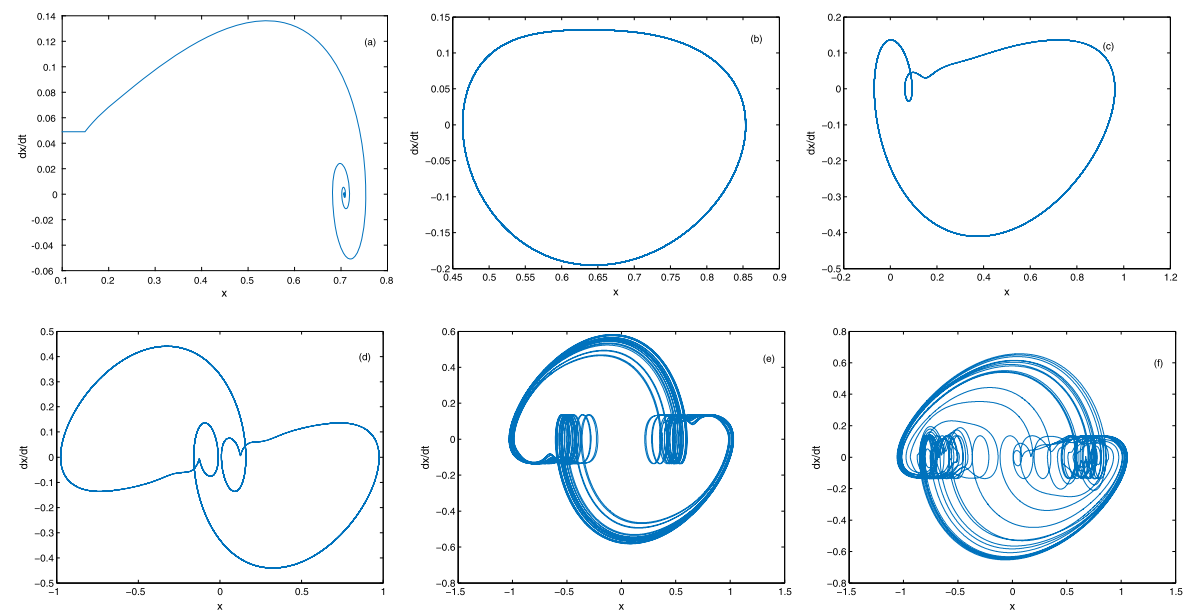

Figure 3 The phase plane plot in $x-d x / d t$ space for different $\tau$ : (a) $\tau=1$ (stability), (b) $\tau=1.8$ (period-1), (c) $\tau=2.6$ (period-2), (d) $\tau=2.7$ (period-4), (e) $\tau=3.1$ (chaos), (f) $\tau=3.3$ (chaos) with parameter $\gamma=0.5$

system becomes divergent at $\tau=1.72$. A phase plane representation in the representative $x-d x / d t$ plane for different $\tau$ is shown in Fig. 5 , which shows the following characteristics: stability $(\tau=0.5)$, period-1 $(\tau=0.9)$, period-2 $(\tau=1.3)$, period $-4(\tau=1.35)$, and chaos $(\tau=1.55$ and $\tau=1.65)$.

\subsection{The dynamic behavior with $\gamma=1.5$}

In the following, we vary the time delay $\tau$ taking $\gamma=1.5$ and $\beta=1$. When $\tau \geq 0.52$, the fixed point loses its stability through Hopf bifurcation, and the bifurcation diagram is shown in Fig. 6. According to the numerical simulations and Fig. 6, the following conclusions are found. The system is asymptotically stable for $\tau \in[0,0.52)$. The limit cycle of period- 1 becomes unstable and a period- 2 cycle appears at $\tau=0.82$. Further period doubling occurs at $\tau=0.88$ (period- 2 to period-4). Through a period doubling sequence, 


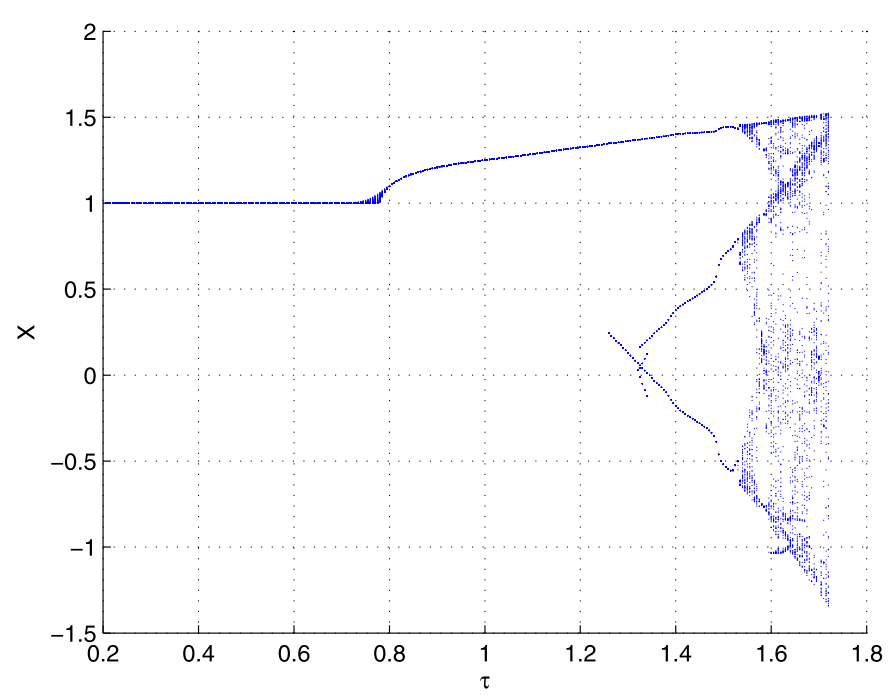

Figure 4 The bifurcation diagram of system (17) with the parameter $\gamma=1$
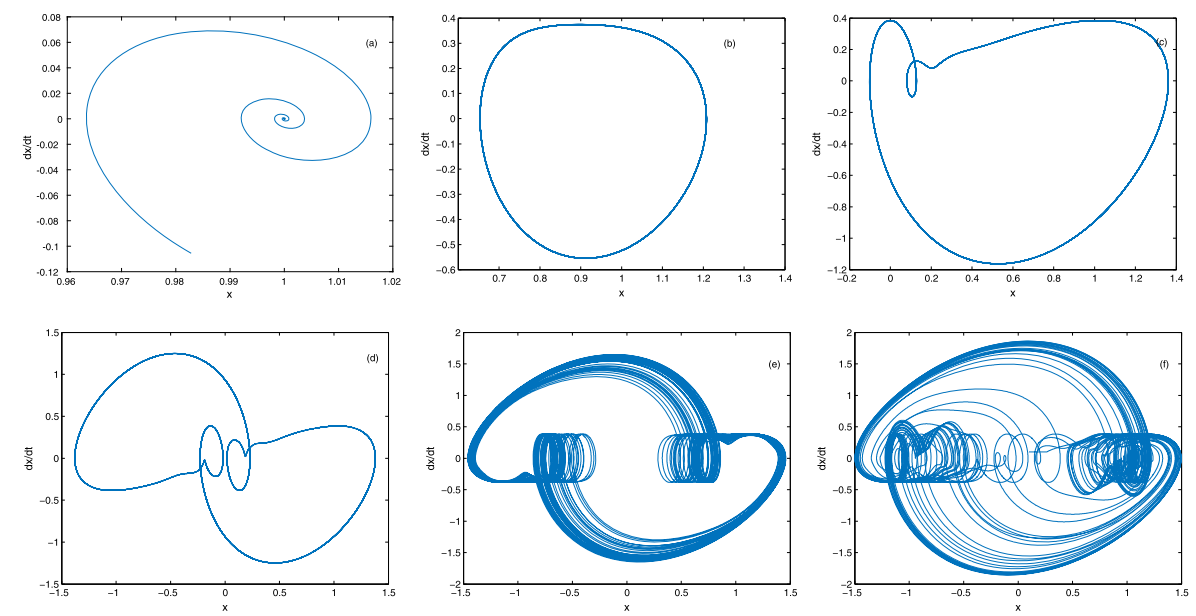

Figure 5 The phase plane plot in $x-d x / d t$ space for different $\tau$ : (a) $\tau=0.5$ (stability), (b) $\tau=0.9$ (period-1), (c) $\tau=1.3$ (period-2), (d) $\tau=1.35$ (period-4), (e) $\tau=1.55$ (chaos), (f) $\tau=1.65$ (chaos) with parameter $\gamma=1$

the system enters into the chaotic regime at $\tau=1.01$. Then, the system enters into the divergent region at $\tau=1.15$. A phase plane representation in the representative $x-d x / d t$ plane for different $\tau$ is shown in Fig. 7, which shows the following characteristics: stability ( $\tau=0.3)$, period-1 $(\tau=0.6)$, period-2 $(\tau=0.87)$, period-4 $(\tau=0.9)$, and chaos $(\tau=1.03$ and $\tau=1.1)$.

\subsection{The dynamic behavior with $\gamma=2$}

Then we study the dynamic behaviors of system (2) by varying the time delay $\tau$ and taking $\gamma=2$ and $\beta=1$. We find that the fixed point loses its stability through Hopf bifurcation when $\tau \geq 0.39$, and the bifurcation diagram is shown in Fig. 8. According to the numerical simulations and Fig. 8, the following conclusions are found. The system is asymptotically stable for $\tau \in[0,0.39)$. And the limit cycle of period- 1 becomes unstable and a period-2 


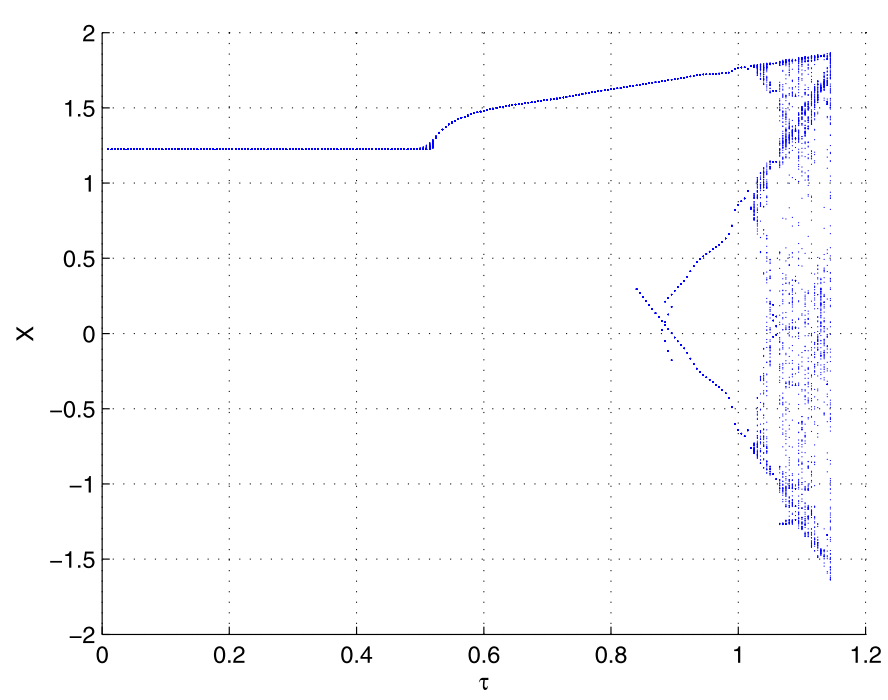

Figure 6 The bifurcation diagram of system (17) with the parameter $\gamma=1.5$
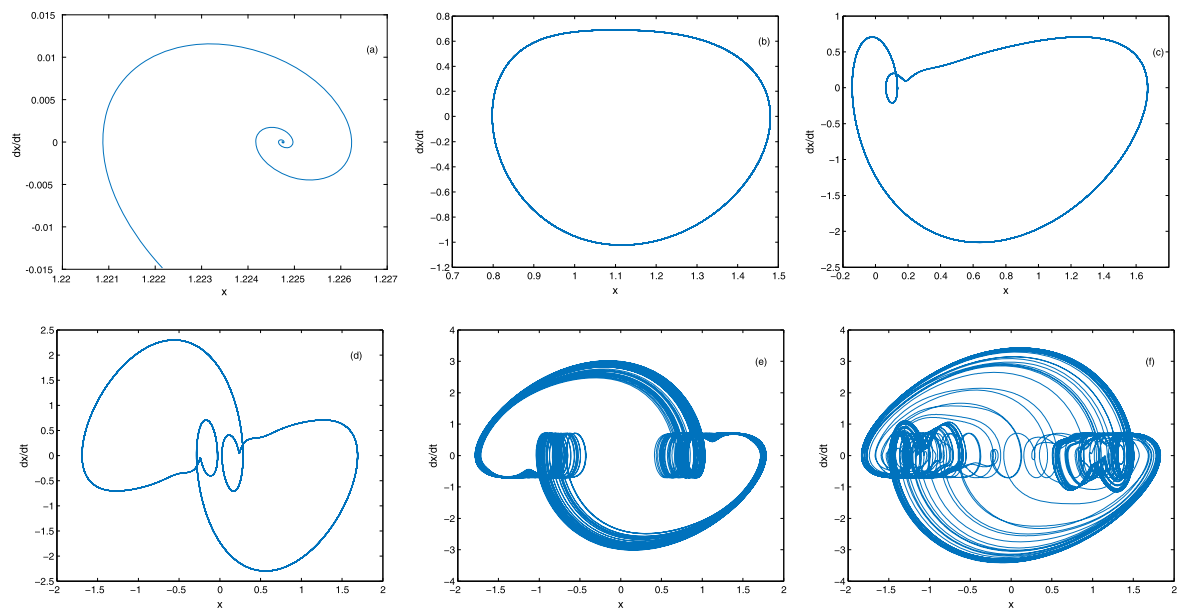

Figure 7 The phase plane plot in $x-d x / d t$ space for different $\tau$ : (a) $\tau=0.3$ (stability), (b) $\tau=0.6$ (period-1), (c) $\tau=0.87$ (period-2), (d) $\tau=0.9$ (period-4), (e) $\tau=1.03$ (chaos), (f) $\tau=1.1$ (chaos) with parameter $\gamma=1.5$

cycle appears at $\tau=0.64$. Further period doubling occurs at $\tau=0.66$ (period- 2 to period4). Through a period doubling sequence, the system enters into the chaotic region at $\tau=$ 0.76 . At last, the system becomes divergent at $\tau=0.86$. A phase plane representation in the representative $x-d x / d t$ plane for different $\tau$ is shown in Fig. 9, which shows the following characteristics: stability $(\tau=0.25)$, period $-1(\tau=0.45)$, period $-2(\tau=0.65)$, period $-4(\tau=$ $0.68)$, and chaos ( $\tau=0.77$ and $\tau=0.83)$.

\subsection{The dynamic behavior with $\gamma=2.5$}

In the following, we study the dynamic behaviors of system (2) when we vary the time delay $\tau$ taking $\gamma=2.5$ and $\beta=1$. It is found that when $\tau \geq 0.31$, the fixed point loses its stability through Hopf bifurcation, and the bifurcation diagram is shown in Fig. 10. According to the numerical simulations and Fig. 10, the following conclusions are found. The system 


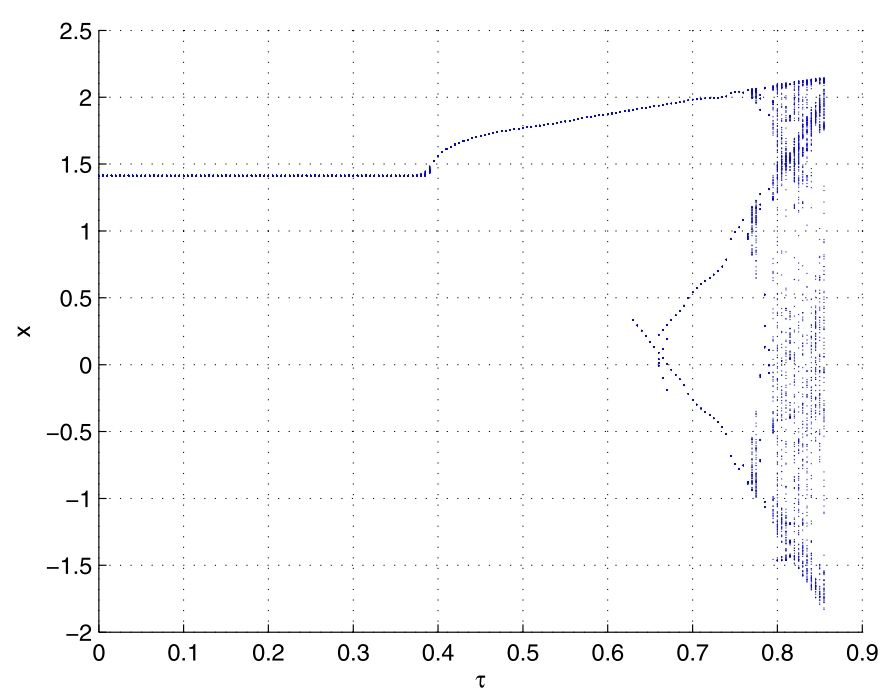

Figure 8 The bifurcation diagram of system (17) with the parameter $\gamma=2$
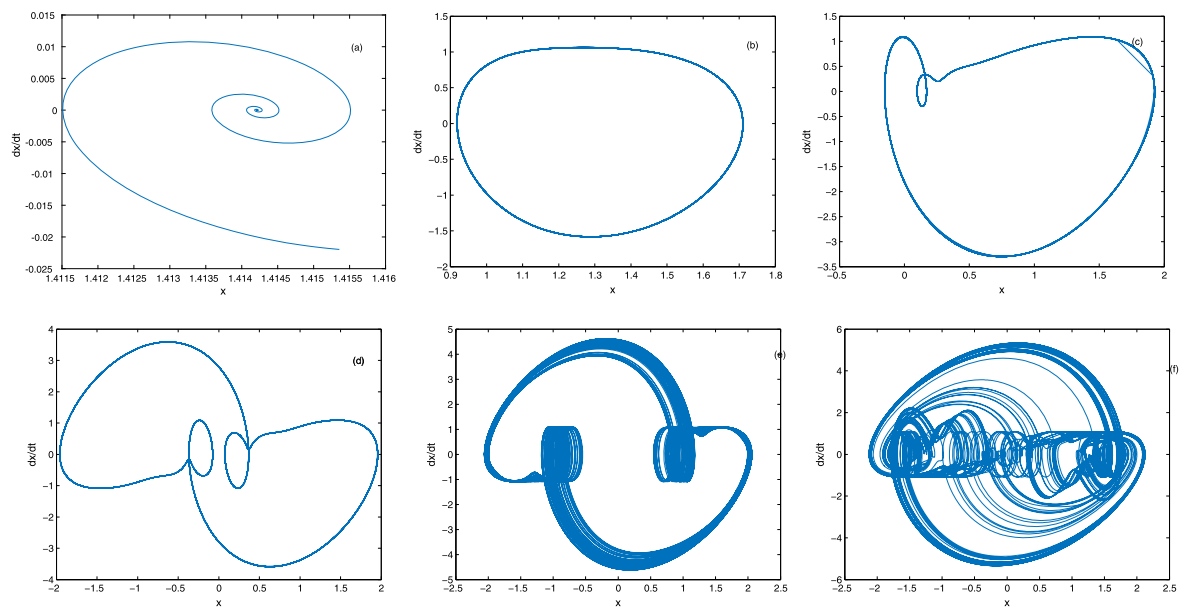

Figure 9 The phase plane plot in $x-d x / d t$ space for different $\tau$ : (a) $\tau=0.25$ (stability), (b) $\tau=0.45$ (period-1), (c) $\tau=0.65$ (period-2), (d) $\tau=0.68$ (period-4), (e) $\tau=0.77$ (chaos), (f) $\tau=0.83$ (chaos) with parameter $\gamma=2$

is asymptotically stable for $\tau \in[0,0.31)$. And the limit cycle of period- 1 becomes unstable and a period- 2 cycle appears at $\tau=0.51$. Further period doubling occurs at $\tau=0.53$ (period-2 to period-4). Through a period doubling sequence, the system enters into the chaotic region at $\tau=0.61$. Then, the system becomes divergent at $\tau=0.69$. A phase plane representation in the representative $x-d x / d t$ plane for different $\tau$ is shown in Fig. 11, which shows the following characteristics: stability $(\tau=0.2)$, period-1 $(\tau=0.36)$, period-2 ( $\tau=0.52)$, period $-4(\tau=0.54)$, and chaos $(\tau=0.62$ and $\tau=0.66)$.

\subsection{The dynamic behavior with $\gamma=3$}

In the end, we study the dynamic behavior of system (2) when we vary the time delay $\tau$ taking $\gamma=3$ and $\beta=1$. When $\tau \geq 0.26$, the fixed point loses its stability through Hopf 


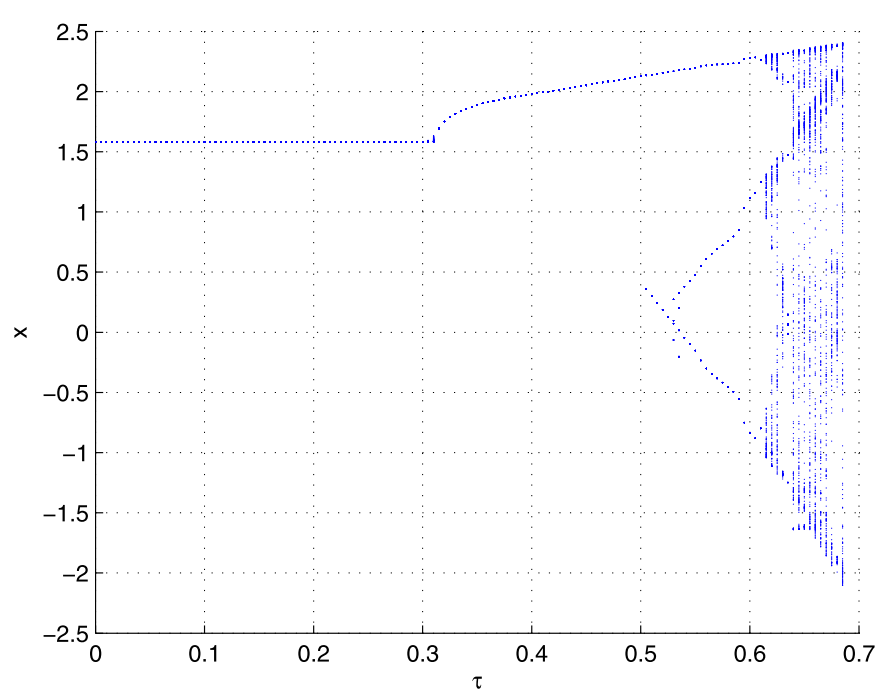

Figure 10 The bifurcation diagram of system (17) with the parameter $\gamma=2.5$
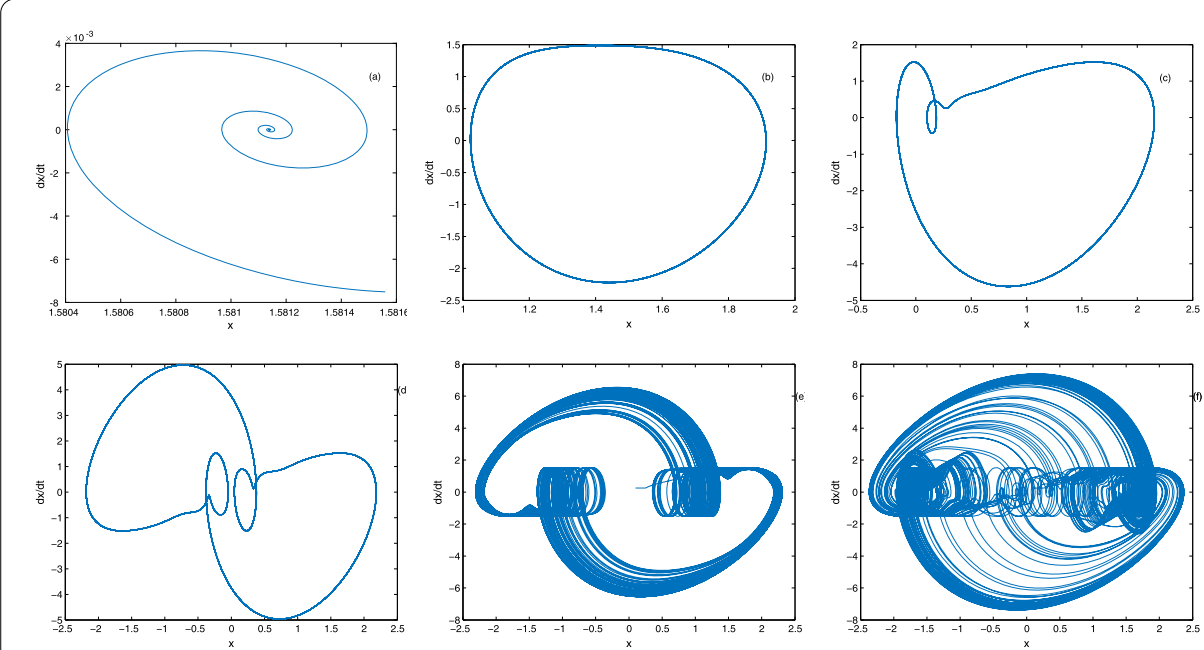

Figure 11 The phase plane plot in $x-d x / d t$ space for different $\tau$ : (a) $\tau=0.2$ (stability), (b) $\tau=0.36$ (period-1), (c) $\tau=0.52$ (period-2), (d) $\tau=0.54$ (period-4), (e) $\tau=0.62$ (chaos), (f) $\tau=0.66$ (chaos) with parameter $\gamma=2.5$

bifurcation, and the bifurcation diagram is shown in Fig. 12. According to the numerical simulations and Fig. 12, the following conclusions are found. The system is asymptotically stable for $\tau \in[0,0.26)$. And the limit cycle of period-1 becomes unstable and a period- $2 \mathrm{cy}-$ cle appears at $\tau=0.42$. Further period doubling occurs at $\tau=0.43$ (period-2 to period-4). Through a period doubling sequence, the system enters into the chaotic region at $\tau=0.51$. Then, the system enters into the divergent region at $\tau=0.57$. A phase plane representation in the representative $x-d x / d t$ plane for different $\tau$ is shown in Fig. 13, which shows the following characteristics: stability $(\tau=0.17)$, period-1 $(\tau=0.3)$, period-2 $(\tau=0.43)$, period-4 $(\tau=0.46)$, and chaos $(\tau=0.52$ and $\tau=0.55)$. 


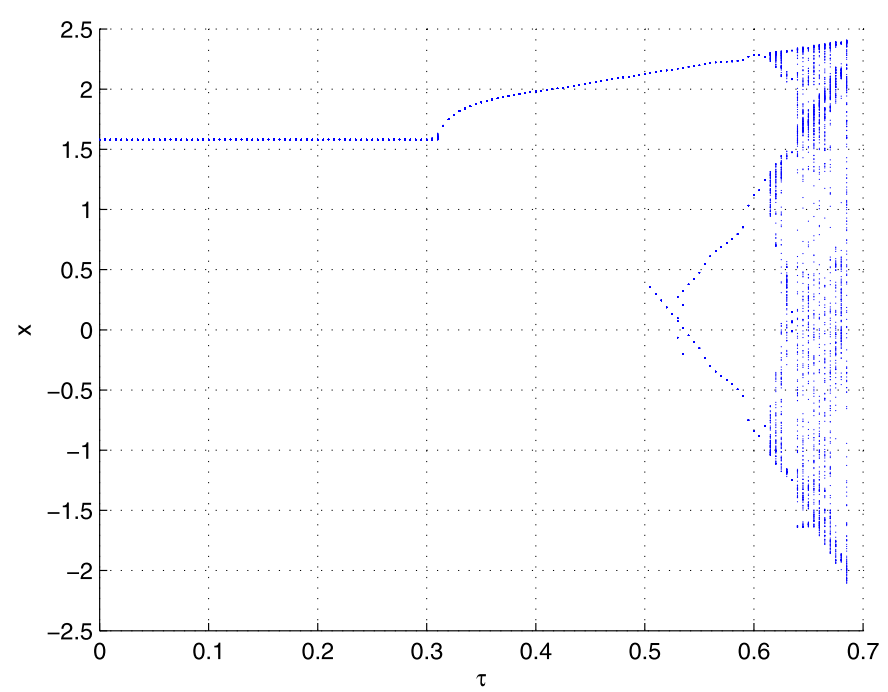

Figure 12 The bifurcation diagram of system (17) with the parameter $\gamma=3$
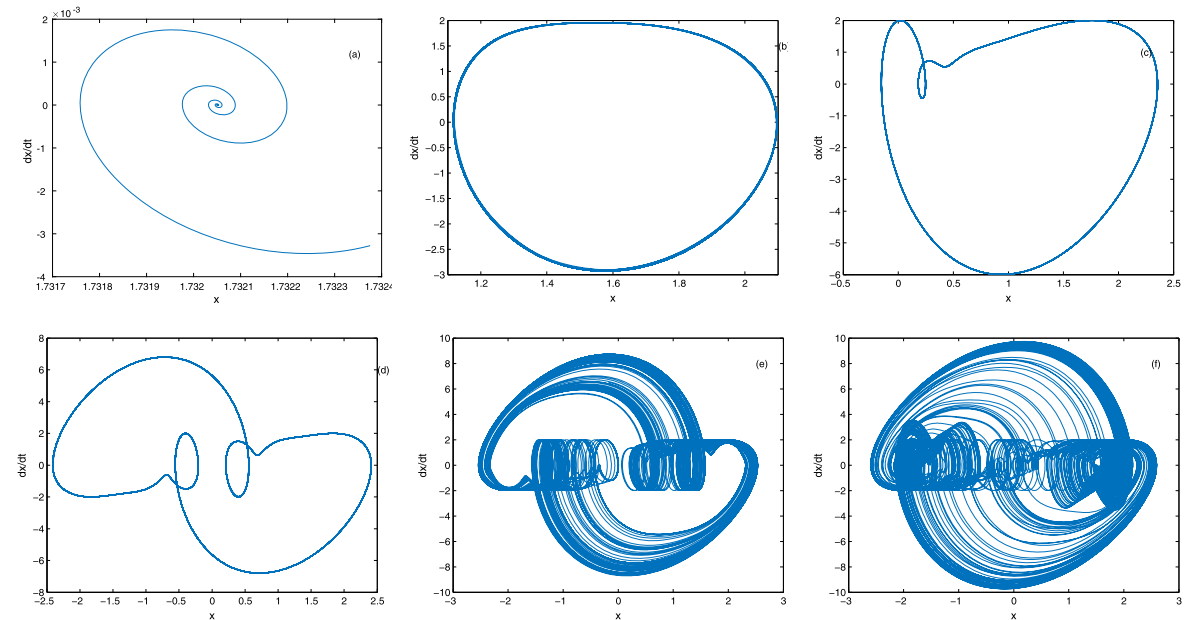

Figure 13 The phase plane plot in $x-d x / d t$ space for different $\tau$ : (a) $\tau=0.17$ (stability), (b) $\tau=0.3$ (period-1), (c) $\tau=0.43$ (period-2), (d) $\tau=0.46$ (period-4), (e) $\tau=0.52$ (chaos), (f) $\tau=0.55$ (chaos) with parameter $\gamma=3$

4.7 The dynamical behavior of the system over the whole $\tau-\gamma$ parameter space

Using the bifurcation analysis theory and the results of numerical simulation, the following conclusions are found: (i) the system is asymptotically stable for $\tau \in[0, \pi /(4 \gamma)$ ); (ii) the system has periodic solutions when $\tau \in(\pi /(4 \gamma), 6.08 /(4 \gamma))$; (iii) the system has chaotic behaviors when $\tau \in(6.08 /(4 \gamma), 6.88 /(4 \gamma))$; (iv) the system is divergent when $\tau>6.88 /(4 \gamma)$. The results are given in Fig. 14. The regions (I)-(IV) indicate the stable, periodic, chaotic, and divergent zones of the system, respectively.

\section{Conclusions}

In this paper, the stability and bifurcation of a first-order time-delayed chaotic system are studied. The stability study and bifurcation analysis established that the system shows a supercritical Hopf bifurcation giving birth to a stable limit cycle oscillation. Next, we use 


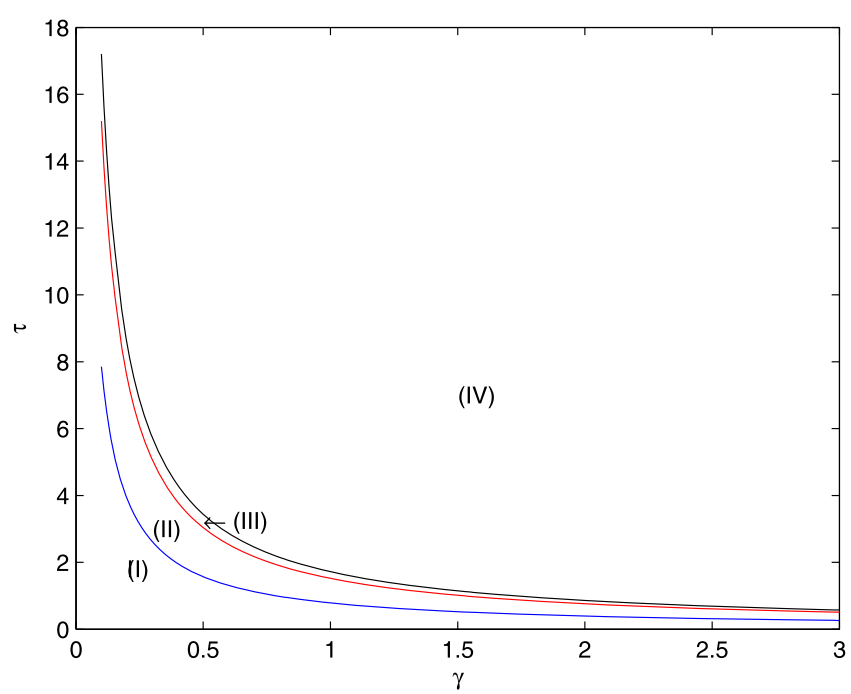

Figure 14 The different regions in the $\tau-\gamma$ parameter space with parameter $\beta=1$

numerical simulations of the prototype system to demonstrate the validity of our method. The numerical simulations with the variation of time delay prove that the system shows a period-doubling route to chaos. And we study the dynamical behaviors of the system over the whole $\tau-\gamma$ parameter space. Then, we give four different regions (I)-(IV) where the system shows stable, periodic, chaotic, and divergent behavior, respectively. In the future work, we will study the limit cycles and homoclinic/heteroclinic orbits in our system.

\section{Funding}

This work is supported by Natural Science Foundation of China (Grant No. 11701397, 61573010), Sichuan Youth Science \& Technology Foundation (No. 2016JQ0046), Artificial Intelligence Key Laboratory of Sichuan Province (No. 2016RYJ06), Fund of Sichuan University of Science and Engineering (No. 2016RCL33, 2015RC10), Opening Project of Key Laboratory of Higher Education of Sichuan Province for Enterprise Informationalization and Internet of Things (No. 2016WYJ04).

\section{Competing interests}

The authors declare that they have no competing interests.

\section{Authors' contributions}

The main idea of this paper was proposed by TL. YW and XZ prepared the manuscript initially and performed all the steps of the proofs in this reserch. All authors read and approved the final manuscript.

\section{Author details}

${ }^{1}$ School of Mathematics and Statistics, Sichuan University of Science and Engineering, Zigong, China. ${ }^{2}$ Artificial Intelligence Key Laboratory of Sichuan Province, Zigong, China. ${ }^{3}$ Key Laboratory of Higher Education of Sichuan Province for Enterprise Informationalization and Internet of Things, Zigong, China. ${ }^{4}$ Yellow River Conservancy Technical Institute, Kaifeng, China.

\section{Publisher's Note}

Springer Nature remains neutral with regard to jurisdictional claims in published maps and institutional affiliations.

Received: 29 July 2018 Accepted: 1 November 2018 Published online: 27 February 2019

\section{References}

1. Wang, Z:: A numerical method for delayed fractional-order differential equations. J. Appl. Math. 2013, 2560711-2560717 (2013)

2. Wang, Z., Huang, X., Zhou, J.P.: A numerical method for delayed fractional-order differential equations: based on G-L definition. Appl. Math. Inf. Sci. 7(2), 525-529 (2013)

3. Liu, L.D., Meng, X.Z.: Optimal harvesting control and dynamics of two species stochastic model with delays. Adv. Differ. Equ. 2017, 18 1-18 (2017) 
4. Li, Y., Zhang, W.H., Liu, X.K.: Stability of nonlinear stochastic discrete-time systems. J. Appl. Math. 2013, 3567461-3567468 (2013)

5. Mackey, M.C., Glass, L.: Oscillation and chaos in physiological control system. Science 197, 287-289 (1977)

6. Ikeda, K., Daido, H., Akimoto, O.: Optical turbulence: chaotic behavior of transmitted light from a ring cavity. Phys. Rev. Lett. 45, 709-712 (1980)

7. Wei, J., Yu, C.: Stability and bifurcation analysis in the cross-coupled laser model with delay. Nonlinear Dyn. 66, 29-38 (2011)

8. Pei, Y., Li, S., Li, C.: Effect of delay on a predator-prey model with parasitic infection. Nonlinear Dyn. 63, 311-321 (2011)

9. Pei, L., Wang, Q., Shi, H.: Bifurcation dynamics of the modified physiological model of artificial pancreas with insulin secretion delay. Nonlinear Dyn. 63, 417-427 (2011)

10. Boutle, I. Taylor, R.H.S., Romer, R.A. El Niño and the delayed action oscillator. Am. J. Phys. 75, 15-24 (2007)

11. Driver, R.D.: A neutral system with state dependent delay. J. Differ. Equ. 54, 73-86 (1984)

12. Liao, X., Guo, S., Li, C.: Stability and bifurcation analysis in tri-neuron model with time delay. Nonlinear Dyn. 49 319-345 (2007)

13. Le, L.B., Konishi, K., Hara, N.: Design and experimental verification of multiple delay feedback control for time delay nonlinear oscillators. Nonlinear Dyn. 67, 1407-1418 (2012)

14. Kwon, O.M., Park, J.H., Lee, S.M.: Secure communication based on chaotic synchronization via interval time varying delay feedback control. Nonlinear Dyn. 63, 239-252 (2011)

15. Li, G., Ling, W.Z., Ding, C.M.: A new comparison principle for impulsive functional differential equations. Discrete Dyn. Nat. Soc. 10, 1-6 (2015)

16. Li, Y.X., Huang, X., Song, Y.W., Lin, J.N.: A new fourth-order memristive chaotic system and its generation. Int. J. Bifurc. Chaos 25(11), 1550151 (2015)

17. Banerjee, T: Single amplifier biquad based inductor-free Chua's circuit. Nonlinear Dyn. 68, 565-573 (2012)

18. Perez, G., Cerdeira, H.: Extracting messages masked by chaos. Phys. Rev. Lett. 74, 1970-1973 (1995)

19. Li, T.Z., Wang, Y., Luo, M.K.: Control of fractional chaotic and hyperchaotic systems based on a fractional order controller. Chin. Phys. B 23, $0805011-08050113$ (2014)

20. Peng, J.H., Ding, E.J., Ding, M., Yang, W.: Synchronizing hyperchaos with a scalar transmitted signal. Phys. Rev. Lett. 76, 904-907 (1996)

21. Li, T.Z., Wang, Y., Yang, Y.: Synchronization of fractional-order hyperchaotic systems via fractional-order controllers. Discrete Dyn. Nat. Soc. 2014, 4089721-40897214 (2014)

22. Li, T.Z., Wang, Y., Yang, Y: Designing synchronization schemes for fractional-order chaotic system via a single state fractional-order controller. Optik 125, 6700-6705 (2014)

23. Kye, W.H., Choi, M., Kurdoglyan, M.S., Kim, C.M., Park, Y.J.: Synchronization of chaotic oscillators due to common delay time modulation. Phys. Rev. E 70, 0462111 (2004)

24. Wang, Y., Li, T.Z.: Synchronization of fractional order complex dynamical networks. Physica A 428, 1-12 (2015)

25. Ando, B., Graziani, S.: Stochastic Resonance: Theory and Applications. Kluwer, Norwell (2000)

26. Fortuna, L., Frasca, M., Rizzo, A.: Chaotic pulse position modulation to improve the efficiency of sonar sensors. IEEE Trans. Instrum. Meas. 52, 1809-1814 (2003)

27. Buscarino, A., Fortuna, A., Frasca, M., Muscato, G.: Chaos does help motion control. Int. J. Bifurc. Chaos 17, 3577-3581 (2007)

28. Wang, Z., Huang, X., Shi, G.D.: Analysis of nonlinear dynamics and chaos in a fractional order financial system with time delay. Comput. Math. Appl. 62(3), 1531-1539 (2011)

29. Campbella, S., Ncubeb, I.: Stability in a scalar differential equation with multiple, distributed time delays. J. Math. Anal. Appl. 450, 1104-1122 (2017)

30. El-Dessoky, M., Yassen, M., Aly, E.: Bifurcation analysis and chaos control in Shimizu-Morioka chaotic system with delayed feedback. Appl. Math. Comput. 243, 283-297 (2014)

31. Song, Y., Han, Y., Zhang, T.: Stability and Hopf bifurcation in a model of gene expression with distributed time delays. Appl. Math. Comput. 243, 398-412 (2014)

32. Feng, Y., Wei, Z:: Delayed feedback control and bifurcation analysis of the generalized Sprott B system with hidden attractors. Eur. Phys. J. Spec. Top. 224, 1619-1636 (2015)

33. Atay, F., Ruan, H.: Symmetry analysis of coupled scalar systems under time delay. Nonlinearity $28,795-824$ (2015)

34. Yeniceri, R., Yalcin, M.: Multi-scroll chaotic attractors from a generalized time-delay sampled-data system. Int. J. Circuit Theory Appl. 44, 1263-1276 (2016)

35. Wei, J., Fan, D.: Hopf bifurcation analysis in a Mackey-Glass system. Int. J. Bifurc. Chaos 17, 2149-2157 (2007)

36. Wei, J., Li, M.: Hopf bifurcation analysis in a delayed Nicholson blowflies equation. Nonlinear Anal., Theory Methods Appl. 60, 1351-1367 (2005)

37. Wei, J., Yuan, Y: Synchronized Hopf bifurcation analysis in a neural network model with delays. J. Math. Anal. Appl. $312,205-229(2005)$

38. Dormaer, P.: Smooth symmetry breaking bifurcation for functional differential equations. Differ. Integral Equ. 5, 831-854 (1992)

39. Dormaer, P.: Smooth bifurcation of symmetric periodic solutions of functional differential equations. J. Differ. Equ. 82, 109-155 (1989)

40. Wu, J.: Symmetric functional differential equations and neural networks with memory. Trans. Am. Math. Soc. 350 4799-4838 (1998)

41. Ruan, S. Wei, J. Periodic solutions of planar systems with two delays. Proc. R. Soc. Edinb. 129A 1017-1032 (1999)

42. Song, Y., Wei, J.: Local Hopf bifurcation and global existence of periodic solutions in a delayed predator-prey system. J. Math. Anal. Appl. 301, 1-21 (2005)

43. Wen, X., Wang, Z.: The existence of periodic solutions for some model with delay. Nonlinear Anal., Real World Appl. 3 , 567-581 (2002)

44. Tang, L.K., Lu, J.A., Lu, J.H., Wu, X.Q.: Bifurcation analysis of synchronized regions in complex dynamical networks with coupling delay. Int. J. Bifurc. Chaos 24(1), 14500111-145001113 (2014)

45. Xiao, M., Zheng, W.X., Jiang, G.P., Cao, J.D.: Stability and bifurcation of delayed fractional-order dual congestion control algorithms. IEEE Trans. Autom. Control 4(2), 361-369 (2017) 
46. Mackey, M.C., Glass, L.: From Clocks to Chaos: The Rhythms of Life. Princeton University Press, Princeton (1988)

47. Ikeda, K., Matsumoto, K.: High-dimensional chaotic behavior in systems with time-delayed feedback. Physica D 29, $223-235$ (1987)

48. Lu, H., He, Z.: Chaotic behavior in first-order autonomous continuous-time systems with delay. IEEE Trans. Circuits Syst. 43, 700-702 (1996)

49. Baker, T.H., Paul, C.A.H., Wille, D.R.: Issues in the numerical solution of evolutionary delay differential equations. Adv. Comput. Math. 3, 171-196 (1995)

50. Baker, T.H., Paul, C.A.H., Wille, D.R.: A bibliography on the numerical solution of delay differential equation. Numerical Analysis Report No. 269, Mathematics Department, University of Manchester, UK (1995)

51. Ucar, A.: On the chaotic behaviour of a prototype delayed dynamical system. Chaos Solitons Fractals 16, 187-194 (2003)

52. Ucar, A.: A prototype model for chaos studies. Int. J. Eng. Sci. 40, 251-258 (2002)

53. Dieudonné, J.: Foundations of Modern Analysis. Academic Press, New York (1960)

54. Lloyd, A.R.J.: Seakeeping: Ship Behaviour in Rough Weather. Ellis Horwood, Chichester (1989)

55. Wei, J.: Bifurcation analysis in a scalar delay differential equation. Nonlinearity 20, 2483-2498 (2007)

56. Hale, J.: Theory of Functional Differential Equations. Springer, New York (1977)

\section{Submit your manuscript to a SpringerOpen ${ }^{\circ}$ journal and benefit from:}

- Convenient online submission

Rigorous peer review

- Open access: articles freely available online

- High visibility within the field

- Retaining the copyright to your article

Submit your next manuscript at $\gg$ springeropen.com 